SANTOS, R.O. et al. Shunt portossistêmico em pequenos animais. PUBVET, Londrina, V. 8, N. 18, Ed. 267, Art. 1781, Setembro, 2014.

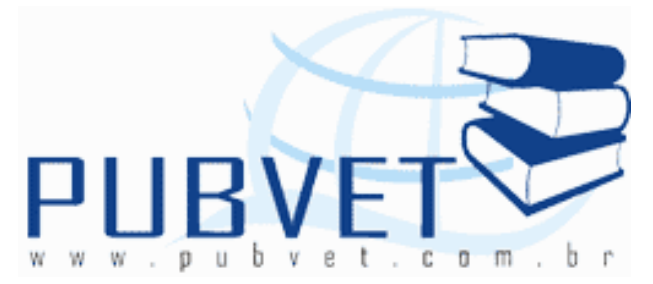

PUBVET, Publicações em Medicina Veterinária e Zootecnia.

\title{
Shunt portossistêmico em pequenos animais
}

Robson Oliveira dos Santos ${ }^{1}$ Carlos Alberto Sanchez ${ }^{1}$, Rogério Carletti Rocha ${ }^{1}$, Michel Eduardo Mello ${ }^{1}$, Angélica Rocio Carvalho ${ }^{2}$

${ }^{1}$ Médico veterinário autônomo

${ }^{2}$ Profa Doutora, diretora do curso de medicina veterinária da Universidade Guarulhos

\section{Resumo}

O desvio portossistêmico (DPS) ou shunt portossistêmico é a anomalia circulatória hepática mais comum em cães. Esta patologia é uma conexão anormal entre a circulação portal e sistêmica que desvia o fluxo sanguíneo do fígado em variados graus. Deste modo, substâncias tóxicas e hepatotróficas importantes oriundas do pâncreas e dos intestinos são absorvidas e enviadas diretamente para circulação, sem passar pelo fígado. Esse decréscimo do fluxo sanguíneo vai resultar em atrofia e subsequente disfunção do fígado, diminuindo cada vez mais o metabolismo hepático das toxinas intestinais que se acumulam no sangue. O tratamento definitivo é cirúrgico, por meio da correção da anomalia vascular por meio de ligadura ou implante de anel 
SANTOS, R.O. et al. Shunt portossistêmico em pequenos animais. PUBVET, Londrina, V. 8, N. 18, Ed. 267, Art. 1781, Setembro, 2014.

metálico. Se a cirurgia não for realizada, o tratamento clínico a longo prazo pode ser eficiente por até dois anos.

Palavras-chave: Encefalopatia hepática, Shunt, Desvio portossistêmico, Anel ameróide.

\title{
Portosystemic shunt in small animals
}

\begin{abstract}
The porto-systemic bypass (DPS) or porto-systemic shunt is the most common hepatic circulatory failure in dogs. This pathology is an abnormal connection between the portal and systemic circulation that diverts blood flow from the liver to varying degrees. Thus, toxic and hepatotróficas important arising from the pancreas and intestines are absorbed and sent directly to circulation, bypassing the liver. This decrease in blood flow will result in subsequent atrophy and dysfunction of liver, steadily decreasing hepatic metabolism of the intestinal toxins that accumulate in the blood. The definitive treatment is surgical, by correcting the anomaly. If surgery is not performed, the long-term clinical treatment can be effective for up to two years.
\end{abstract}

Keywords: hepatic encephalopathy, Shunt, Deviation portosystemic ameroid ring.

\section{Introdução}

O fígado é o maior e um dos mais importantes órgãos de secreção e excreção do corpo (CENTER, 1992). Recebe a maior parte do seu sangue da veia porta e uma porção menor através da artéria hepática (JUNQUEIRA, 2008).

O desvio portossistêmico (DPS) ou shunt porto-sistêmico é a anomalia circulatória hepática mais comum em cães. Esta patologia é uma conexão anormal entre a circulação portal e sistêmica que desvia o fluxo sanguíneo do fígado em variados graus. Portanto, o sangue proveniente dos órgãos abdominais que deveria ser drenado pela veia porta em direção ao fígado, 
SANTOS, R.O. et al. Shunt portossistêmico em pequenos animais. PUBVET, Londrina, V. 8, N. 18, Ed. 267, Art. 1781, Setembro, 2014.

sofre um desvio e flui parcialmente para outra veia de grande importância sistêmica (como veia cava caudal, ou veia ázigos ou outros vasos sistêmicos), entrando na circulação sistêmica. Deste modo, as substâncias tóxicas (amônia, metionina / mercaptanas, ácidos graxos de cadeia curta, ácidos $\gamma$ aminobutíricos) absorvidas pelos intestinos e as substâncias hepatotróficas importantes oriundas do pâncreas e dos intestinos são enviadas diretamente para essa circulação, sem passar pelo fígado. Esse decréscimo do fluxo sanguíneo (no qual estão presentes as substâncias hepatotróficas) vai resultar em atrofia e subsequente disfunção do fígado, diminuindo cada vez mais o metabolismo hepático das toxinas intestinais que se acumulam no sangue (DEWEY, 2006; FOSSUM, 2006; BUNCH, 2010).

Essas comunicações podem ser congênitas ou adquiridas (LAMB \& WHITE, 1998; VULGAMOTT, 1979). Também podem ser classificadas como intra ou extra-hepáticas, sendo a intra-hepática decorrente da persistência de fluxo sangüíneo através do ducto venoso, mais comum nas raças de grande porte (LAMB \& WHITE, 1998). Raças "toys" apresentam a forma congênita extrahepática com maior freqüência, porém também há relatos de desvios intrahepáticos (HUNT et al., 2000; FOSSUM, 2006).

O DPS adquirido difere do congênito, pois enquanto a etiologia congênita desenvolve-se na fase embrionária, a adquirida ocorre como uma compensação orgânica, quando houver hipertensão portal decorrente de outras alterações hepáticas como cirrose e hepatite (PELOI, 2012)

O DPS congênito ocorre como resultado da persistência de um vaso fetal, que em condições de normalidade fecha depois do nascimento, ou do desenvolvimento anômalo do sistema venoso vitelino, que origina uma conexão funcional anormal. Após o nascimento a veia umbilical é cortada e a pressão sanguínea e a diminuição de oxigênio promovem o fechamento do 
SANTOS, R.O. et al. Shunt portossistêmico em pequenos animais. PUBVET, Londrina, V. 8, N. 18, Ed. 267, Art. 1781, Setembro, 2014.

ducto venoso (vaso fetal) em um período de dois a seis dias. Quando a oclusão do ducto venoso não ocorre, o sangue proveniente do trato gastrointestinal deixa de passar pelo fígado e ganha acesso imediato à circulação sistêmica (SILVA, 2009).

Os DPS intra-hepáticos são considerados como uma falha no fechamento das comunicações fetais, ductos venosos persistentes, que comunicam a veia umbilical e a cava; Existe a probabilidade que esses tipos de pontes sejam vasos aberrantes. As fístulas arteriovenosas são congênitas e provenientes de dilatações de veias pré-existentes não funcionais ou trombose com comunicações portossistêmicas da veia hepática portal (TOBIAS, 2007). Shunts congênitos intra-hepáticos constituem cerca de $35 \%$ dos desvios individuais em cães e cerca de 10\% em gatos (FOSSUM, 2006).

Os DPS congênitos extra-hepáticos se originam da veia porta, veia gástrica esquerda ou da veia esplênica e se conectam à veia cava caudal (mais comuns), à veia ázigos ou a outros vasos sistêmicos como a torácica interna, a renal e a cólica (menos frequentes) (JOHNSON, 1999). Os DPS congênitos extra-hepáticos são geralmente vasos anômalos únicos e respondem por quase $63 \%$ dos desvios únicos nos cães (FOSSUM, 2006). Um shunt portossistêmico extra-hepático é considerado congênito quando uma veia ou raramente duas veias anormais estão presentes sem sinais de hipertensão portal concomitantes (FAVIER, 2004; SZATMARI, 2004).

O objetivo do presente trabalho é abordar aspectos do quadro de DPS em cães em gatos, focando principalmente nos tratamentos clínicos e cirúrgicos utilizados atualmente em nossa rotina. 
SANTOS, R.O. et al. Shunt portossistêmico em pequenos animais. PUBVET, Londrina, V. 8, N. 18, Ed. 267, Art. 1781, Setembro, 2014.

\section{Sinais Clínicos}

Os sinais clínicos do DPS adquirido são variáveis e estão normalmente relacionados com a degeneração hepática e dificuldade do fígado exercer suas funções normais (PELOI, 2012). Entretanto DPS geralmente são congênitos e são mais comumente diagnosticados em animais menores de um ano de idade (FOSSUM, 2006).

Em estudo realizado entre 1980 e 2001 Tobias (2003) pôde evidenciar após análise de 1.227 .236 cães, que 1.847 cães portadores de DPS e desses 593 eram da raça Yorkshire terriers. Essa raça tem predisposição 20 vezes maior do que todos outros cães combinados TOBIAS (2007).

Os sinais clínicos de DPS congênito extra-hepático são: Sintomas referentes ao sistema nervoso central, gastrointestinal e trato urinário. Os distúrbios gastrintestinais tbm podem ocorrer em casos de doença intestinal inflamatória, obstrução intestinal, ingestão de corpo estranho e exagero dietético (BUNCH, 2010).

Os animais afetados geralmente são avaliados por causa da incapacidade de crescer, pequena estatura corporal, ou perda de peso. Outras anormalidades comuns incluem depressão, ptialismo (especialmente em gatos), amaurose, e alterações comportamentais (FOSSUM, 2006; (BUNCH, 2010). Poliúria e polidipsia são achados comuns, mas não consistentes, já que não são sinais exclusivos de hepatopatias (ROTHUIZEN; MEYER, 2004; FOSSUM, 2006; MEHL et al., 2007). Wrigley (1987) afirma que podem ser encontrados sinais clínicos de depressão ou hiperexcitabilidade, ataxia e cegueira aparente.

Sinais de encefalopatia hepática podem variar muito daqueles que são extremamente leves e difíceis de identificar como uma significativa anormalidade (letargia, cansaço) para alterações graves como, por exemplo: 
SANTOS, R.O. et al. Shunt portossistêmico em pequenos animais. PUBVET, Londrina, V. 8, N. 18, Ed. 267, Art. 1781, Setembro, 2014.

Fraqueza, estupor, convulsões ou coma (FOSSUM, 2006). Contudo esses sinais referentes ao sistema nervoso central podem, também, estar presente em distúrbios infecciosos, como cinomose, intoxicações, distúrbios metabólicos como hipoglicemia, displasia de occiptal e hidrocefalia (TILLEY; SMITH, 2003).

Aspectos inespecíficos de disfunção hepática estão presentes como sinais clínicos do DPS congênito, tais sinais são: anorexia ou polifagia, incluindo, vômito e diarreia intermitente ou constipação. Esses sinais não necessariamente estão acompanhados de encefalopatia hepática (WRIGLEY, 1987; JOHNSON, 2003)

Alguns animais são apresentados para avaliação com disfunções urinárias como: hematúria, disúria, polaquiúria, estrangúria e obstrução uretral. A urolitíase é um sinal comum e está presente em mais de $50 \%$ dos cães com DPS (FOSSUM, 2006). Exceto quando por biurato de amônio, esses sinais são semelhantes aos das afecções do trato urinário (TILLEY; SMITH, 2003).

Os sinais clínicos de hipertensão portal incluem dor, distensão abdominal do íleo ou ascite, diminuição da pressão venosa central, tempo de preenchimento capilar prolongado, mucosas pálidas e pulsos periféricos fracos. O quadro de ascite raramente é visto em cães com DPS congênito a menos que haja grave hipoproteinemia, hemorragia gastrointestinal grave, ou hipertensão portal. Tipicamente, o fluido de qualquer uma destas condições seria claro, transudato puro (WILLARD, 1999, Apud BERENT; TOBIAS, 2009)

Outros achados clínicos menos importantes são: febre intermitente e prurido intenso (ROTHUIZEN; MEYER, 2004). Os rins podem estar proeminentes a palpação. A cor dourada ou cobre da íris tem sido observada em muitos gatos com DPS (FOSSUM, 2006). 
SANTOS, R.O. et al. Shunt portossistêmico em pequenos animais. PUBVET, Londrina, V. 8, N. 18, Ed. 267, Art. 1781, Setembro, 2014.

\section{Diagnóstico e exames complementares}

As doenças hepáticas, em geral, podem ser diagnosticadas com base no exame clínico, testes laboratoriais e exames por imagem. Também são necessárias um conjunto de informações que compreendem anamnese detalhada e exames específicos de avaliação da função hepática (ROTHUIZEN; MEYER, 2004; PELOI, 2012).

A hipoglicemia é frequente em cães com DPS congênito simples extra-hepático e pode ser causada por insuficiência de glicogênio, devido à capacidade inadequada que o fígado apresenta de armazenamento de glicose e metabolização anormal de insulina (HOSKINS, 1997).

O hemograma pode evidenciar microcitose com eritrócitos normocrômicos, células em alvo e anemia arregenerativa leve (MEYER, 1995). Baixos níveis séricos de albumina é um achado comum, no entanto, alguns cães (e a maioria dos gatos) portadores de DPS possuem valores normais de albumina, há reduzida conversão de amônia em uréia (FOSSUM, 2006).

Concentração sérica de ácidos biliares com 12 horas de jejum e 2 horas pós prandial, é o teste laboratorial mais sensível pra avaliar a existência de DPS (ROTHUIZEN et al.,1982). Entretanto Sassaki (2001) esclarece que seu uso não é rotineiro devido ao custo elevado. Entretanto quando esse método for utilizado deve ser associado com os outros resultados laboratoriais e sinais clínicos, pois apenas esse teste não diferencia doença vascular portossistêmica de insuficiência hepática (BUNCH, 1995).

Há evidências de que os ácidos biliares inibem, diretamente, a síntese de colesterol. Hipocolesterolemia pode ser encontrada na maioria dos animais 
SANTOS, R.O. et al. Shunt portossistêmico em pequenos animais. PUBVET, Londrina, V. 8, N. 18, Ed. 267, Art. 1781, Setembro, 2014.

com DPS congênito, mas o colesterol pode estar dentro da normalidade (HOSKINS, 1997).

O teste de tolerância de amônia também é importante indicativo para o diagnóstico do DPS. A amônia, originada principalmente através do catabolismo dos aminoácidos dietéticos, é metabolizada e transformada em uréia no fígado. Em casos de DPS, essa transformação fica prejudicada, o que explica a existência da hiperamonemia, que é uma indicação direta de encefalopatia hepática (HOSKINS, 1997).

Os urólitos encontrados em urinálise são os de biuratos de amônio, devido ao acúmulo de amônia no sangue, que será filtrada nos rins, originando tais cálculos (SASSAKI, 2001). Iso e hipostenúria também são resultados frequentemente observados nesse exame (HUNT, 2000).

Radiografias abdominais são importantes como exame de triagem para DPS congênito. É extremamente raro um cão com DPS que não tem algum grau de microhepatia (FOSSUM, 2006).

A ultrassonografia pode detectar um desvio, mas um exame com resultado normal não descarta a sua presença. Fístulas arteriovenosas hepáticas podem ser diferenciadas de outras desordens portossistêmicas pelo ultrassom com Doppler (NYLAND, 2004).

Kamikawa (2012) observou após estudo ultrassonográfico que em cães portadores de DPS o diâmetro médio e a área média da veia porta, da veia cava caudal e da aorta apresentaram valores superiores aos dos cães não portadores e em região de hilo hepático, o grupo dos cães com shunt, a velocidade média de fluxo sanguíneo portal mediu $22,29 \mathrm{~cm} / \mathrm{s}$, e para o grupo de cães livres da doenças, a velocidade de fluxo sanguíneo portal apresentou- 
SANTOS, R.O. et al. Shunt portossistêmico em pequenos animais. PUBVET, Londrina, V. 8, N. 18, Ed. 267, Art. 1781, Setembro, 2014.

se menor, com média de $17,76 \mathrm{~cm} / \mathrm{s}$. Nesse estudo não foi observada a influência do sexo sobre a morfometria e hemodinâmica vascular.

As vantagens inerentes ao exame ultrassonográfico como a sua disponibilidade, a sua não invisibilidade e a rara necessidade de utilização da anestesia, justificam a sua utilização para pesquisa de shunts portossistêmicos na rotina veterinária ( $D^{\prime}$ ANJOU et al. 2004). A ultrassonografia tornou-se a ferramenta diagnóstica de escolha para DPS. Tanto desvios intra como extrahepáticos foram identificadas através dessa técnica (FOSSUM, 2006).

Pode-se observar turbilhonamento do fluxo sanguíneo no local de inserção da vascularização anômala na veia cava caudal ao ultrassom com doppler (Kamikawa, 2012).

O diagnóstico definitivo de DPS é feito através de portografia contrastada, identificação do shunt através de ultrassonografia, cintilografia hepática nuclear ou identificação do desvio em laparotomia exploratória. A técnica de portografia contrastada é o método mais simples e mais eficaz (FOSSUM, 2006; PELOI, 2012). Entretanto, se disponível, a cintilografia ainda é reconhecida como o procedimento não invasivo mais confiável para a documentação da existência de desvios portossistêmicos (NYLAND, 2004).

\section{Tratamento}

O tratamento definitivo é cirúrgico, por meio da correção da anomalia vascular, porém alguns pacientes podem apresentar sinais neurológicos graves e de difícil controle, mesmo após a correção. A cirurgia é contraindicada na presença de hipertensão portal (VULGAMOTT, 1979).

Todos os animais com DPS são considerados candidatos para cirurgia (TOBIAS, 2007). Entretanto Peloi (2012) afirma que não há correção cirúrgica para 
SANTOS, R.O. et al. Shunt portossistêmico em pequenos animais. PUBVET, Londrina, V. 8, N. 18, Ed. 267, Art. 1781, Setembro, 2014.

shunt portossitêmico adquirido, esses pacientes devem ser tratados apenas clinicamente, sendo o prognóstico reservado, onde alguns conseguem qualidade de vida em longo prazo.

Antes de submeter o animal ao procedimento cirúrgico é muito importante estar atento a suas condições, assim, deve-se iniciar um tratamento clínico para correção de líquidos, taxa de glicemia, ganho de peso, estado geral do animal e, principalmente, controle de encefalopatia hepática, esse tratamento é feito por alguns dias até que se o animal tenha condições de ser submetido a um protocolo anestésico e, então, à cirurgia (BUNCH, 2010; TOBIAS, 2007). Deve-se ter muito cuidado com a anestesia, já que muitos fármacos anestésicos são metabolizados pelo fígado, que se apresenta insuficiente em casos de anomalia vascular portossistêmica. Assim, a metabolização dessas drogas fica, acentuadamente reduzida (BUTLER et al., 1990).

A complicação mais comum da manobra cirúrgica é a hipertensão portal, que ocorre quando se faz uma ligação total do vaso anômalo. Ocorre, pois, a vasculatura intra-hepática ainda não é capaz de suportar um grande fluxo sanguíneo que passa a chegar ao fígado (MURPHY, 2001).

A técnica cirúrgica deve ser realizada com o objetivo de ligar o vaso anômalo para, então, corrigir o desvio de fluxo sanguíneo. Atualmente, ao invés de se fazer a ligação do vaso através de fios de sutura, utilizam-se constritores ameróides (MURPHY, 2001). Outra técnica utilizada atualmente para ocluir o vaso anômalo é a utilização de faixa de papel celofane envolvendo o vaso. Fazse um anel, com o papel celofane, em torno dele (MEHL, 2007).

A ligadura ou a atenuação do shunt pode ser extremamente desafiadora porque muitas vezes o vaso é de difícil localização. Ocasionalmente, os shunts podem ser identificados como depressões palpáveis em um lóbulo do fígado, 
SANTOS, R.O. et al. Shunt portossistêmico em pequenos animais. PUBVET, Londrina, V. 8, N. 18, Ed. 267, Art. 1781, Setembro, 2014.

ou ele pode ser visto entrando na veia cava caudal se ela não está completamente rodeada por tecido parenquimatoso (FOSSUM, 2006). É importante que, em qualquer técnica escolhida, a oclusão do vaso seja gradual (MEHL et al, 2005, apud NETO, 2013).

O mecanismo de funcionamento do anel constritor depende de uma substância higroscópica, a substância ameróide, que se encontra na parte interna do anel. Essa substância, então absorve líquido lentamente e, assim, a parte interna no anel aumenta seu volume e faz a constrição do vaso, já que o diâmetro do lúmen central diminui devido à expansão da substância ameróide. A oclusão ocorre à medida que vai ocorrendo fibrose ao redor do vaso. A parte externa do anel é composta por um anel de aço inoxidável, com uma fenda que permite colocá-lo ao redor do vaso; há uma chave para impedir que ele se desprenda do vaso (KYLES et al., 2002).

Após a oclusão parcial do vaso, as vísceras devem ser observadas, por alguns minutos, a fim de avaliar a ocorrência de cianose e congestão, indicativos de aumento de pressão portal (TOBIAS, 2007). Kamikawa (2012) em estudo hemodinâmico através de ultrassonografia não observou sinais de hipertensão portal no período pós-cirúrgico em cães com DPS submetidos ao tratamento cirúrgico a partir da colocação do anel ameróide.

A morbidade pós-cirúrgica é causada pela hipoglicemia, a mortalidade cirúrgica é baixa em casos de desvios extra-hepáticos simples, na maior parte dos casos, a melhora clínica ocorre no primeiro dia pós-cirúrgico (TOBIAS, 2007).

Há estudos que revelam que, de dois a quatro meses após a cirurgia, o fígado do animal se regenera, podendo ter um aumento de 43 a $62 \%$. Esses dados foram evidenciados a partir de tomografia computadorizada (STIEGER et al., 2007, apud NETO, 2013). 
SANTOS, R.O. et al. Shunt portossistêmico em pequenos animais. PUBVET, Londrina, V. 8, N. 18, Ed. 267, Art. 1781, Setembro, 2014.

Após a cirurgia, o tratamento clínico também deve ser feito até que ocorra a melhora das funções hepáticas (HOSKINS, 1997). Esse tratamento é paliativo e visa o controle da encefalopatia e insuficiência hepática. A terapia medicamentosa se consiste em antibióticos (metronidazol ou neomicina) para reduzir a população bacteriana produtora de uréase e de lactulose, que aumenta a eliminação do conteúdo intestinal e acidifica seu lúmen, e da dieta hipoprotéica, que oferece menos substrato para a produção de amônia no intestino (BUNCH, 2010).

A dieta hipoprotéica também ajuda na não formação dos uratos, a urina deve ser alcalinizada para não haver a precipitação desses cristais (NETO, 2009). Entretanto (BUNCH, 2007) afirma que pelo fato de o DPS congênito ser comum em animais em fase de crescimento, não é recomendada a restrição proteica por um período longo antes da cirurgia, isso se houver indicação cirúrgica.

O tratamento clínico a longo prazo pode ser eficiente por até dois anos em alguns casos, mas isso depende da gravidade dos sintomas e de acordo com a localização do vaso anômalo. Sabe-se que muitos cães tratados clinicamente, por muito tempo, não ficam normais completamente, apresentando sinais neurológicos refratários, pois o tratamento clínico não é capaz de reverter a microhepatia e as alterações no metabolismo de carboidratos, lipídeos e proteínas (BUNCH, 2004).

Terapia de fluido intravenoso para substituir e manter a hidratação é necessário se um animal apresenta reclinada e incapacidade de beber água, ou desidratação devido a perda de fluidos por gastroenterites. Suplementação de potássio é muitas vezes necessária devido à depleção de potássio em cães com diarreia crônica, hipocalemia também pode contribuir para encefalopatia hepática (BROME 2004). 
SANTOS, R.O. et al. Shunt portossistêmico em pequenos animais. PUBVET, Londrina, V. 8, N. 18, Ed. 267, Art. 1781, Setembro, 2014.

Os episódios eméticos são tratados suspendendo- se a alimentação, o animal é mantido na fluidoterapia, aplica-se cimetidina ou ranitidina, pois há aumento de secreção gástrica, metoclopramida é usada como antiemético (TABOADA, 1990).

Os probióticos estão sendo utilizados para combater a hiperamonemia em pacientes humanos (LOGUERCIO et al., 1995). Bactérias, como Bifidobacterium bifidum e Streptococcus faecium, diminuem competitivamente a microbiota produtora de urease, reduzindo a absorção de amônia (LOGUERCIO et al., 1987). A lactulose, por sua vez, é considerada um prebiótico, pois aumenta a capacidade lactofermentativa de populações de Lactobacillus. Essa substância altera a metabolização dos aminoácidos, ao agir sobre o funcionamento das bactérias intestinais, pois acidifica o meio, que capta amônia e desestimula sua produção. Funciona, também, como um catártico, já que promove diarreia osmótica (BUNCH, 2004). A utilização de lactulose e frutooligossacarídeos, tem ação sinérgica, na estimulação do crescimento de bactérias benéficas (SCHUMANN, 2002).

Para o controle da hipoglicemia administra-se solução de dextrose a 2,5\% ou $5 \%$, acrescentada a soluções balanceadas de eletrólitos (TOBIAS, 2007).

\section{Considerações Finais}

Desvios e anormalidades vasculares hepáticas não são problemas de vasta ocorrência em nossa rotina. Porém devemos sempre incluí-lo em nosso diagnóstico diferencial. Devido à falta de sinais específicos não podemos abrir mão de métodos diagnósticos que possam confirmar ou descartar a doença. 
SANTOS, R.O. et al. Shunt portossistêmico em pequenos animais. PUBVET, Londrina, V. 8, N. 18, Ed. 267, Art. 1781, Setembro, 2014.

Segundo Peloi (2012) quando trata se de desvios adquiridos pouco se pode fazer. Vulgamott (1979) enfatiza que a cirurgia é contraindicada na presença de hipertensão portal. Enquanto TOBIAS (2007) refere que todos os animais diagnosticados com DPS devem ser submetidos a intervenção cirúrgica. Entretanto levando em consideração que a disseminação da doença está em sua maior parte ligada a animais reprodutores portadores de DPS congênito, devemos imediatamente indicar a retirada desses animais da reprodução, sendo a castração indicada independente se tratamento do desvio seja clinico ou cirúrgico. Tobias (2003) evidenciou que cães da raças Yorkshire Terrier possuem predisposição 35 vezes maior para serem diagnosticados com DPS do que todas as outras raças combinadas. Esta representação é fortemente sugestiva de uma predisposição genética para a doença.

Deve-se atentar no diferencial do DPS adquirido ou congênito, intra ou extra hepático, essa diferenciação é de grande importância para a determinação do tratamento e para prognóstico.

Bunch (2007) referencia que devido à limitada resposta inflamatória, não se recomenda o uso de faixa de celofane em gatos. No caso da utilização do anel ameróide não se recomenda a utilização de anéis com diâmetro menor que $5,0 \mathrm{~mm}$ visto que o desvio adquirido pode ocorrer com maior frequência em pacientes que receberam o implante de tamanho menor.

Bunch (2010) afirma que cães portadores de DPS têm maior prevalência de outros defeitos congênitos, como criptorquidia e distúrbios cardíacos. Acreditasse que isso se dá devido à diminuição do fluxo sanguíneo na veia umbilical quando o animal ainda se encontra no útero. Entretanto podemos observar a necessidade de mais estudos para melhor compreensão da fisiopatologia da doença. 


\section{Referências Bibliográficas}

BERENT A., TOBIAS K. Portosystemic vascular anomalies. Vet. Clin. North Am., Small Anim. Pract. 2009,ed 39:513-541. Disponível em:

http://www.eadveterinaria.com.br/material/270/1972/shunts_2009.pdf. Acesso em: 19 de maio de 2013

BROME C.J., WALSH V.P., BRADDOCK J.A. Shunts portossistêmicos congênitas em cães e gatos. NZ Vet J. 2004; v. 52 p.154-62.

BUNCH, S. E. Diagnosis and management ot portosystemic shunts in dogs and cats. Veterinary previews. A Purina publication for veterinarians, 1995, n. 4, p. 2-6.

BUNCH S.E. Distúrbios hepáticos agudos e sistêmicos que acometem o fígado. In: ETTINGER, S.J.; FELDMAN, A. Tratado de Medicina Interna Veterinária, 5 ed. , São Paulo: Manole, 2004, v. 2. p $1398-1413$.

BUNCH, S.E.; WATSON P. J. , Distúrbios hepatobiliares in: NELSON, R.W.; COUTO, C.G. Medicina interna de pequenos animais. 4 ed., São Paulo: Elsevier, 2010. Cap.38, p.542 - 578.

BUTLER, L. M. et al. Surgical management of extrahepatic portosystemic shunts in the dog and cat. Seminars in veterinary medicine and surgery (small animal), 1990, v. 5, n.2, p. 127-133.

CENTER S.A. Fisiopatologia e diagnóstico laboratorial das moléstias hepáticas. In: ETTINGER, S.J.; FELDMAN, A. Tratado de Medicina Interna Veterinária, 5. ed., São Paulo: Manole, 1992, v. 3. p. $1487-1546$.

D 'ANJOU M.A., PENNINK D., CORNEJO L. \& PIBAROT P. Ultrasonographic diagnosis of portosystemic shunting in: dogs and cats. Vet. Radiol. Ultrasound, 2004, ed 45 p.424-437.

DEWEY, C.W. Encefalopatias distúrbios cerebrais in: Neurologia de Cães e Gatos: Guia prático, São Paulo: Roca, 2006, p. 59 - 99.

FOSSUM, T. W. Intrahepatic shunts: cut or to coil?. In: $30^{\circ}$ World Congress Of The World Small Animal Veterinary Association. Prague, Czech Republic, 2006. Disponível em: $<$ www.ivis.org/proceedings/wsava/2006/lecture25/Fossum8.pdf?LA=1 >. Acesso em: 01 de maio de 2013.

HOSKINS, J.D. O fígado e o pâncreas. In: Pediatria veterinária. 2. ed. Rio de Janeiro: Interlivros, 1997, p. 172.

HUNT, G.B. et al. Congenital portosystemic shunt in: Toy and miniature poodles. Aust Vet J, v.78, n.8, p.530-532, 2000.

JOHNSON, S.E. Desvio Sanguíneo Portossistêmico: Consulta Veterinária em 5 minutos: Espécie Canina e Felina. 3 ed. São Paulo: Manole, 2008.

JUNQUEIRA, L.C.; CARNEIRO, J. Histolgia Básica. 11.ed. Rio de Janeiro: guanabara Koogan, p. 268-284, 2008. 
LAMB, C.R.; WHITE, R.N. Morphology of congenital intrahepatic portacaval shunts in dogs and cats. Vet Rec, 1998 v.17, p.55-59.

LOGUERCIO, C. et al. Enterococcus lactic acid bacteria strain SF 68 and lactulose in hepatic encephalopathy in: a controlled study. J Int Med Res, 1987, v.15, n.6, p.335-343..

LOGUERCIO, C. et al. Long term effects of Enterococcus faecium SF 68 versus lactulose in the treatment of pacients with cirrhosis and grade I-II hepatic encephalopathy. J Hepat,1995 v.23, n.1, p.39-46.

KAMIKAWA L. ; BOMBONATO P.P. Avaliação morfométrica e hemodinâmica comparativa dos vasos envolvidos no shunt portossistêmico congênito em cães. Pesquisa Veterinária Brasileira,2012, 32 ed, p 941-946.

KYLES, A. E. et al. Evaluation of ameroid ring constrictors for the management of single extrahepatic portosystemic shunts in cats in: Journal of the American veterinary medical association, 2002 , v. 220 , p. $1.341-1.347$.

MADDISON, J. E. Canine congenital portosystemic encephalopathy. Australian veterinary Journal,1988, v. 65, n. 8, p. 245-249.

MEHL, M. L. et al. Surgical management of left-divisional intrahepatic portosystemic shunts: outcome after partial ligation of, or ameroid ring constrictor placement on, the left hepatic vein in twenty-eight dogs (1995-2005). Veterinary surgery, 2007, v. 36, p. 21-30.

MEYER, D. J. et al. Testes e distúrbios dos eritrócitos. In: Medicina de laboratório veterinária. São Paulo: 1995, Roca, p. 20.

MURPHY, S. T. A Comparison of the Ameroid Constrictor Versus Ligation in the Surgical Management of Single Extrahepatic Portosystemic Shunts. Journal of the American animal hospital association, 2001, v. 37, p. 390-396.

NETO A.A., BRACCIALLI C.S. Desvio portossistêmico congênito simples extra-hepático em cães in: Unimar Ciências, Marília: Unimar, Vol. 18, p 23 - 31, Disponível em

http://www.unimar.br/publicacoes/2011/unimar_ciencias18.pdf Acesso: 13 de março de 2013

NYLAND T.G. \& MATTOON J.S. 2004. Ultrassom Diagnóstico em Pequenos Animais. 2a ed. Roca, Sao Paulo, 2004. p. 95-129.

PELOI C., MACHADO, T.V.; MOREIRA, P.K. Shunt portossistêmico adquirido, relato de caso in: Pet South American, 2012, Disponível em:

http://www.petsa.com.br/uploads/TrabalhosAprovados/MedicinaInterna/620.pdf , Acesso 30 de abril de 2013

ROTHUIZEN, J. et al. Congenital porto-systemic shunts in sixteen dogs and three cats. Journal of small animal practice,1982, v. 23, p.67-81.

ROTHUIZEN J. , MEYER H.P. Anamnese, exame físico e sinais da doença hepática in: ETTINGER, S.J.; FELDMAN, A. Tratado de Medicina Interna Veterinária, 5. ed., São Paulo: Manole, 2004, v. 2. p.1342-1347. 
SASSAKI, R. A. et al. Desvio porto-sistêmico congênito simples extra hepático: correção com constritor ameróide. Relato de caso. Clínica veterinária,2001, n. 33, p. 27-32.

SCHUMANN, C. Medical, nutritional and technological properties of lactulose. An update, Eur J Nutr, 2002, v. 41, n.1, p.17-25.

SILVA, V.C. et al. Ultrassonografia doppler e angiografia tomográfica computadorizada no diagnostico de desvios portosistemicos. Revisão de Literatura.Clínica veterinária,2009, n.78, p. 70-78.

SZATMARI V., Standard planes for ultrasonographic examination of the portal system in dogs. J. Am. Vet. Med. Assoc. 2004, v. 224, p 713-716.

TABOADA, J. Medical management of animals with portosystemic shunts. Seminars in veterinary medicine and surgery (small animal), 1990, v. 5, n.2, p. 107-119.

TILLEY L. P., SMITH F. W. K. Encefalopatia hepática in: Consulta veterinária em 5 minutos. Espécies canina e felina. 2 ed. São Paulo: Manole, 2003.

TOBIAS K.M., Determination of inheritance of single congenital portosystemic shunts in Yorkshire Terriers, in: Journal of the American Animal Hospital Association, 2003, 39 ed. p.385-389

TOBIAS K.M. in: Desvios portossistêmicos e outras anomalias vasculares hepáticas in: SLATTER D. Manual de cirurgia de pequenos animais. V.1, 3 ed. Barueri, SP: Manole, 2007, p. 727-751.

WILLARD M.D., TWEDT H. Doenças Gastrointestinais, do pâncreas, e hepáticas. In: WILLARD M.D., TWEDT H., TURNWALD G.H., Diagnóstico clínico de pequenos animais por métodos laboratoriais. 3 ed. Philadelphia: WB Saunders Company, 1999, p. 172-207.

WRIGLEY, R.; KONDE, L.J.; PARK, R.D; LEBEL, J.L. Ultrasonographic diagnosis of portocaval shunts in: Young dogs. Journal of the American Veterinary medical association, 1987, v. 191, n.4, p.421-424.

VULGAMOTT, J.C. Hepatic encephalopathy associated with acquired portacaval shunt in a dog. J Am Vet Med Assoc, 1979, v.175, n.7, p.724-726. 